\title{
Laparoscopic treatment of symptomatic non-ruptured proximal and middle segment splenic artery aneurysm
}

\author{
(1) Ender Anılır, (1) Şenol Carıllı, (1) Aydın Alper \\ Department of General Surgery, İstanbul American Hospital, İstanbul, Turkey
}

\begin{abstract}
A splenic artery aneurysm is the third most frequent intraabdominal aneurysm, accounting for $60 \%$ of all visceral artery aneurysms. There is a rupture rate of $2 \%$ with an associated mortality rate of $36 \%$. Treatment of splenic artery aneurysm may include laparotomy, laparoscopy, or endovascular techniques. Excellent results have been reported with laparoscopic techniques recently. Presently described is a case of symptomatic, non-ruptured proximal and middle segment splenic artery aneurysm treated with laparoscopic surgery. A 27-year-old female patient applied to the general surgery polyclinic complaining of abdominal pain and dyspepsia. After evaluating all of the clinical findings, a diagnosis of splenic artery aneurysm was made and a laparoscopic splenectomy and aneurysmectomy were performed. The patient was discharged on postoperative day 8 . During the postoperative follow-up period, she recovered very well with no clinical problem.
\end{abstract}

Keywords: Aneurysm; laparoscopy; non-rupture; splenic artery.

\section{Introduction}

Splenic arter aneurysm (SAAs) account for $60 \%$ of all visceral artery aneurysms. SAAs are the third most frequent intraabdominal aneurysms, following abdominal aorta and iliac artery aneurysms with an estimated prevalence of $0.8 \%$ in the population. ${ }^{[1-4]}$ Most occur in the distal third of the splenic artery (75\%) followed by the middle third (20\%) whereas the proximal splenic artery aneurysms are uncommon. ${ }^{[5]}$ Although the pathophysiology of Splenic artery aneurysm (SAA) remains unclear, a number of conditions, including atherosclerosis, medial degeneration, abdominal trauma, hyperflow conditions (e.g., pregnancy and portal hypertension), connective tissue disorders, vasculitis may contribute to the development of disease. ${ }^{[6]}$ İts incidence is four times higher in women than in men. ${ }^{[4]}$ SAAs may be complicated by rupture in $2-10 \%$ of cases but risk of rupture with mortality approaching $40 \%$.

${ }^{[7]}$ Treatment of SAA includes laparotomy, laparoscopy or endovascular techniques. ${ }^{[5]}$ Although few cases of SAA in the proximal splenic artery treated by laparoscopic techniques have been reported in the literature, laparoscopic aneurysmectomy with or without spleen preservation has been reported with excellent results recently. ${ }^{[5,8]}$

We aimed to present symptomatic non-ruptured proximal and middle segment splenic artery aneurysm case treated by laparoscopic surgery.

\section{Case Report}

The 27-year-old female patient had applied to the general surgery polyclinic complaining of abdominal pain from 


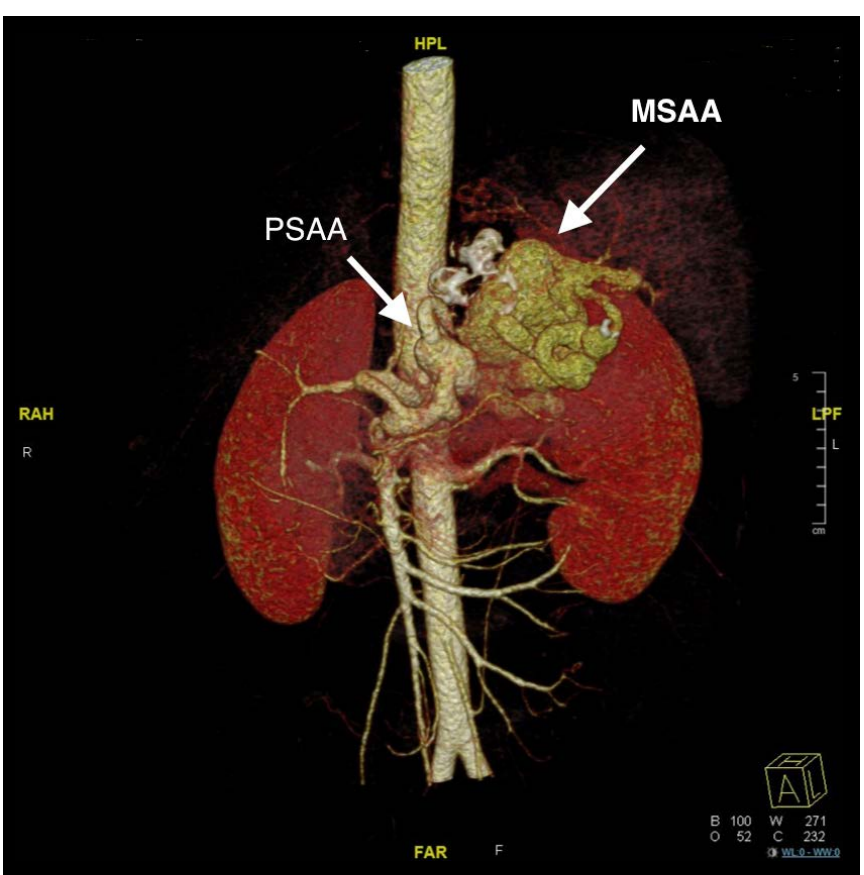

Figure 1. Multiple aneurysms in splenic artery on CT (MSAA: Middle SAA; PSAA: Proximal SAA).

time to time and dyspepsia occurs after dinner especially. The patient had not previously undergone abdominal surgery. She was taking steroid and cyclophosphamide because of Behçet Disease and SAA for 3 years. She didn't ease about abdominal pain in spite of taking drugs. She didn't have illness history of her family. Blood pressure was 120/80; pulse was $80 / \mathrm{min}$. There was no fever. There was neither abdominal tenderness nor palpable mass. Intestinal sounds were normoactive; rectal examination was normal. There was no significant finding in hemogram and biochemistry test results.

Computed tomography (CT) scan (Fig. 1) and three-dimensional CT angiography (Fig. 2) demonstrated the presence of multiple aneurysms in splenic artery, maximum diameter was 33x47x60 mm and includes partial thrombosis. Moreover, there was high degree stenosis in proximal splenic artery segment shortly before celiac trunk. Considering the anatomic location in a long segment artery of the aneurysm and its size, endovascular treatment was not proposed due to recanalization and coil migration. Laparoscopic ligation of the splenic artery (SA) combined with splenectomy was indicated as optimal treatment option.

After appropriate perioperative preparation, the procedure was done under general anesthesia, with patient being positioned in the supine position. Four trocars were inserted: $10 \mathrm{~mm}$ trocar (para-umbilical as camera port), $5 \mathrm{~mm}$ two trocars on the right upper qaudrant and

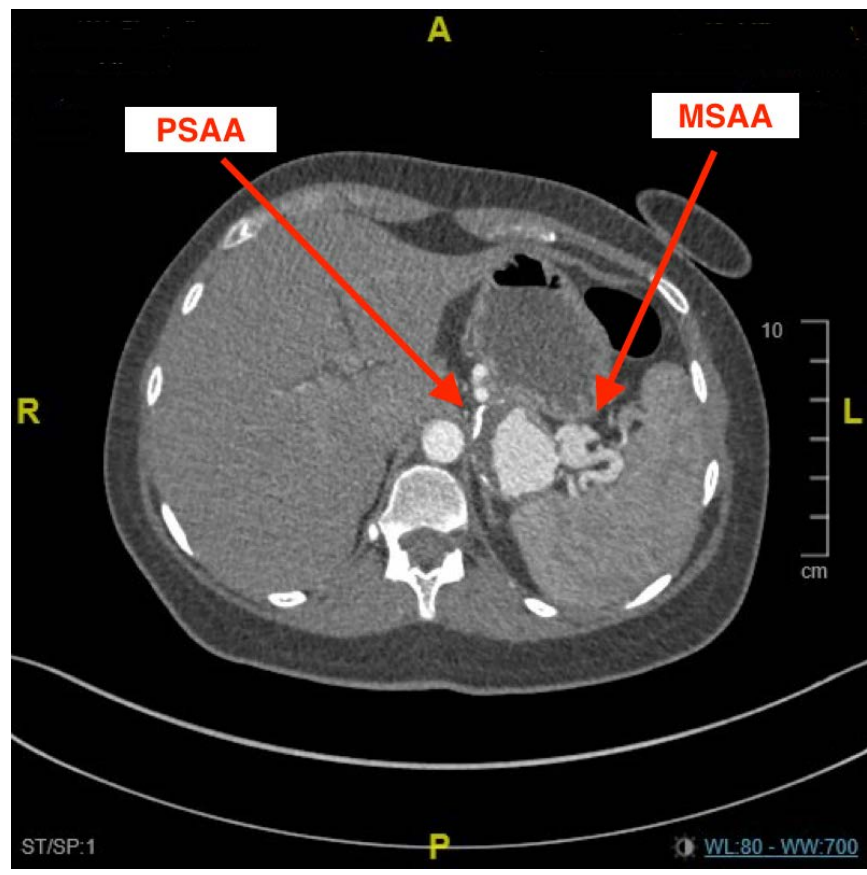

Figure 2. Multiple aneurysms in three-dimensional CT angiography (MSAA: Middle SAA; PSAA: Proximal SAA).

hypocondrium, $5 \mathrm{~mm}$ only one trocar on the left hypocondrium. Pneumoperitoneum was created. First, the gastrocolic ligament was divided, revealing the pancreatic edge, then the splenic artery identified and the aneurysm was exposed before cutting by endoscopic ligasure and harmonic. Multiple aneurysms were identified, the proximal one was the largest. Dissection was kept going from splenic hilum till celiac trunk. We separated aneurysm's proximal and distal parts, and L hemoloc clips were used to ligate the aneurysm proximally and distally. The whole spleen appeared dusky when we visualised. Gastrosplenic ligament was divided and splenic flexura was dissected. After we completed splenectomy and separeting vessel segments from surrounding tissues, specimen was taken out from abdomen in endobag (Fig. 3a-e). The abdominal cavity was irrigated with normal saline and a drain was placed relative operation area to drain and observe the intraabdominal fluid and prevent contingent complications caused by pancreatic leakage. The operative time was 110 minutes and blood loss was $300 \mathrm{~mL}$. The patient had a smooth recovery with no complications and was discharged on postoperative day 8. During the postoperative follow-up period, she recovered very well without neither abdominal pain nor pancreatic insufficiency, as well as whole wound healing. She completed postoperative long term period in 2 months smoothly. Maximum aneurysm diameter was $5 \times 4 \times 2.5 \mathrm{~cm}$ and multiple true aneurysms occured in proximal and middle artery segments, however, 

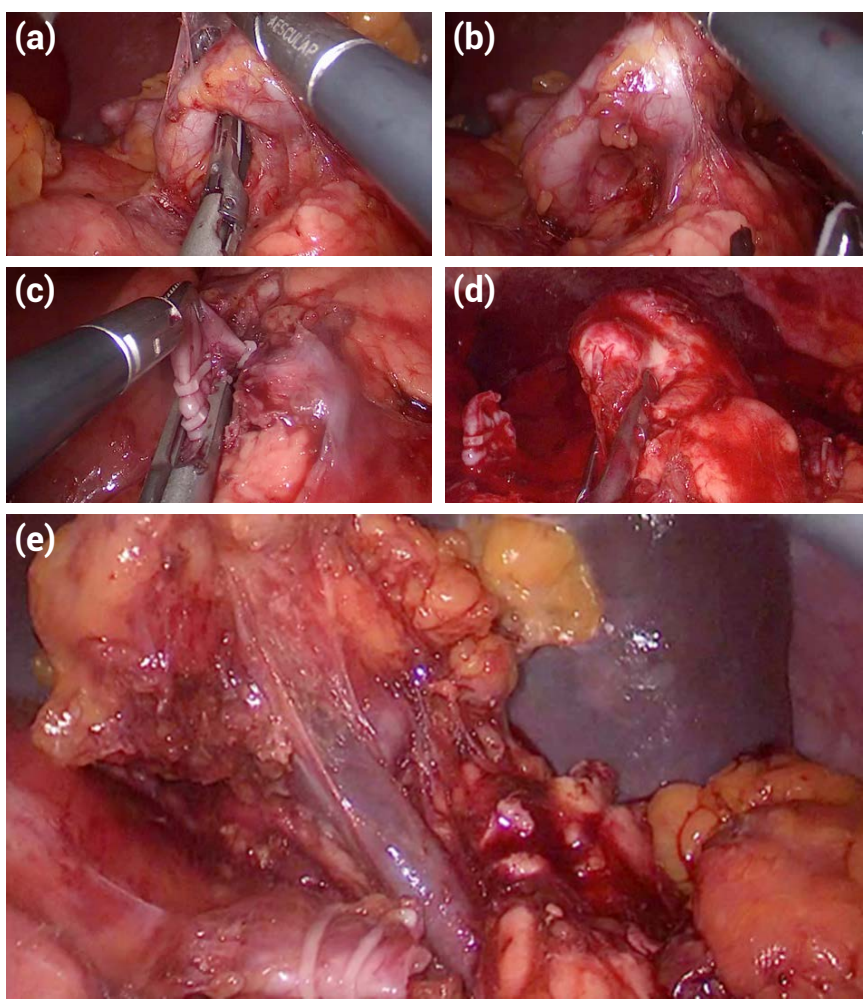

Figure 3. (a) Dissection of middle splenic artery aneurysm. (b) Releasing surrounding tissues from artery. (c) Ligating splenic artery close to celiac trunk. (d) Aneurysm after dividing from celiac trunk. (e) Splenic vein after dividing and cutting aneurysm.

it wasn't in rapport with vasculitis in pathology report of resected material.

\section{Discussion}

Splenic artery aneurysm treatment method is defined by both etiologic factors, size and location of artery, in the same way, rupture risk is desicive in determination for surgery or endovasculary approach. Splenic artery aneurysms are mostly asymptomatic and are discovered incidentally. On the contrary, symptoms as epigastric and left-upper quadrant abdominal pain, dyspepsia might be related to either big dimension and location of aneurysm or risk of rupture. ${ }^{[2,7]}$ Rupture risk is increased with pregnancy, portal hypertension, liver transplantation, and concomitant vasculitis and aneurysms larger than $2 \mathrm{~cm}$ in diameter. ${ }^{[8-10]}$ In our case, rupture risk, due to aneurysm approximately $4 \mathrm{~cm}$ in diameter and concomitant Behçet's Disease, was effective cause for surgery desicion. If we think, aneurysm rupture rate of $2 \%$ with an associated mortality rate of $36 \%$; it may be said that, true splenic artery aneurysms are rare but potentially life threatening entities. Any untreated aneurysm will progress in size and will eventually rupture..$^{[7,8]}$
The treatment options for SAAs depend on aneurysm dimension, location and severity of the clinical findings. The most frequent treatment options for SAAs are open abdominal surgery, endovascular treatment (coil embolization or stent), laparoscopic surgery, which is becoming more popular, and medical treatment. ${ }^{[2,11,12]}$

Although transcatheter arterial embolization is widely accepted as a suitable and less invasive procedure for unruptured visceral artery aneurysms, it is occasionally impossible for certain reasons, e.g., allergy to contrast agent or technical problems. Also, splenic abscess formation, splenic infarct, high rate of recurrence and inability to perform in larger lesions are disadvantages for endovascular technique. In such situations, a surgical approach is a necessary and reliable procedure. İn addition to this, laparoscopic surgery has become increasingly accepted as a surgical approach for unruptured visceral artery aneurysms. ${ }^{[7,11]}$ In recent years, open aneurysm repair of SAAs has been largely replaced by minimally invasive surgery which result in less surgical trauma and faster postoperative recovery. Thus, laparoscopy may be a challenging alternative, not only to endovascular procedures, but also open surgery. On the other hand, it is contraindicated in hemodynamically unstable patients or those at rupture risk and not suitable for larger aneurysms and lesions with dense adhesions to surrounding tissues inspite of the fact that laparoscopic excision can be the optimal treatment, particularly in early pregnancy and with small lesions safely. ${ }^{[2,5]}$ Laparoscopic SAA treatment techniques as, aneurysm excision, ligation, aneurysmectomy plus splenectomy, differ in accordance with aneurysm features etc. location, size. ${ }^{[13]}$ Generally, aneurysmectomy and reconstruction is an option for proximal SAA, while distal SAA requires aneurysmectomy with splenectomy, and sometimes it requires even distal pancreatectomy if the aneurysm is too closely adherent to the tail of pancreas. ${ }^{[12]}$ We preferred splenectomy plus artery resection without any distal pancreas resection in our case due to aneurysm approximately $4 \mathrm{~cm}$ dimention and longish proximal plus middle segment location.

For a successful laparoscopic treatment of splenic artery aneurysms, it is essential to be proficient in advanced laparoscopy, since the dissection of the aneurysm can be very difficult and risky. Sudden rupture of the thin wall of the aneurysm should always be feared during its dissection off the surrounding tissue. Special attention should be paid to avoid damaging the splenic vein. İt is important 
to control of the splenic artery proximal and distal to the aneurysm for safety. Splenic artery runs separate from the pancreatic parenchyma, and a plane can always be found between the two tissues so as to preserve pancreas from injury. Laparoscopy has more advantages instead of open surgery owing to the gentle exposure of the laparoscopic approach, with avoidance of retractors and of direct manipulation of the pancreas. ${ }^{[14]}$ We didn't have any trouble about dissection surrounding vessels and pancreas in our case.

\section{Conclusion}

Laparoscopic treatment of SAA is safety and effective. It has more advantages than open surgery and endovascular technique. Specifying appropriate patients for laparoscopy and to be proficient in advanced laparoscopy is essential for achievement of technique.

\section{Disclosures}

Informed Consent: Written informed consent was obtained from the patient for the publication of the case report and the accompanying images.

Peer-review: Externally peer-reviewed.

Conflict of Interest: None declared.

\section{References}

1. Uyar is, Okur FF, Akpınar B, Abacılar F, Yurtman V, Şahin V, et al. A giant splenic artery aneurysm: a case report. Turk Gogus Kalp Damar Cerrahisi Dergisi 2013;21:799-802.

2. Akbulut S, Otan E. Management of Giant Splenic Artery Aneurysm: Comprehensive Literature Review. Medicine (Baltimore) 2015;94:e1016.

3. Stanley JC, Thompson NW, Fry WJ. Splanchnic artery aneurysms. Arch Surg 1970 Dec;101:689-97.
4. Tcbc-Rj RA, Ferreira MC, Ferreira DA, Ferreira AG, Ramos FO. Splenic artery aneurysm. [Article in English, Portuguese]. Rev Col Bras Cir 2016;43:398-400.

5. Wei YH, Xu JW, Shen HP, Zhang GL, Ajoodhea H, Zhang RC, et al. Laparoscopic ligation of proximal splenic artery aneurysm with splenic function preservation. World $\mathrm{J}$ Gastroenterol 2014;20:4835-8.

6. Qiu P, Zhu H. Endovascular Repair of Anomalous Splenic Artery Aneurysm with Systemic Lupus Erythematosus. Ann Vasc Surg 2019;55:309.e1-309.e4.

7. Yadav S, Sharma P, Singh PK, Punia S, Desai P, Anjan AK, et al. Giant splenic artery aneurysm: A rare but potentially catastrophic surgical challenge. Int J Surg Case Rep 2012;3:5336.

8. Hosn MA, Xu J, Sharafuddin M, Corson JD. Visceral Artery Aneurysms: Decision Making and Treatment Options in the New Era of Minimally Invasive and Endovascular Surgery. Int J Angiol 2019;28:11-6.

9. De Silva WSL, Gamlaksha DS, Jayasekara DP, Rajamanthri SD. A splenic artery aneurysm presenting with multiple episodes of upper gastrointestinal bleeding: a case report. J Med Case Rep 2017;11:123.

10. Dolar E, Uslusoy H, Kiyici M, Gurel S, Nak SG, Gulten M, et al. Rupture of the splenic arterial aneurysm due to Behcet's disease. Rheumatology (Oxford) 2005;44:1327-8.

11. Toyoda $Y$, Igami T, Ochiai Y, Ebata T, Yokoyama Y, Sugawara $\mathrm{G}$, et al. Single-Incision Laparoscopic Splenectomy for an Unruptured Aneurysm of the Splenic Artery. Med Princ Pract 2018;27:95-8.

12. Martin $D$, Teixeira Farinha $H$, Dattner $N$, Rotman $S$, Demartines $\mathrm{N}$, Sauvain MO. Spontaneous non-traumatic splenic artery aneurysm rupture: a case report and review of the literature. Eur Rev Med Pharmacol Sci 2018;22:3147-50.

13. Nasser HA, Kansoun AH, Sleiman YA, Mendes VM, Van Vyve E, Kachi A, et al. Different laparoscopic treatment modalities for splenic artery aneurysms: about 3 cases with review of the literature. Acta Chir Belg 2018;118:212-8.

14. Pietrabissa A, Ferrari M, Berchiolli R, Morelli L, Pugliese $\mathrm{L}$, Ferrari V, et al. Laparoscopic treatment of splenic artery aneurysms. J Vasc Surg 2009;50:275-9. 